Relations industrielles

Industrial Relations

\title{
Notion, objectifs, possibilités
}

\section{Jacques St-Laurent}

Volume 24, numéro 1, 1969

URI : https://id.erudit.org/iderudit/027985ar

DOI : https://doi.org/10.7202/027985ar

Aller au sommaire du numéro

Éditeur(s)

Département des relations industrielles de l'Université Laval

ISSN

0034-379X (imprimé)

1703-8138 (numérique)

Découvrir la revue

Citer cet article

St-Laurent, J. (1969). Notion, objectifs, possibilités. Relations industrielles /

Industrial Relations, 24(1), 57-63. https://doi.org/10.7202/027985ar
Résumé de l'article

Après avoir défini la notion de politique de salaires, l'auteur tente d'en expliquer les objectifs et d'identifier les moyens à employer pour la rendre efficace. Mais, en fait, on parle beaucoup de politique de salaires mais que fait-on concrètement à ce sujet ?
Tous droits réservés (C Département des relations industrielles de l'Université Laval, 1969
Ce document est protégé par la loi sur le droit d'auteur. L’utilisation des services d'Érudit (y compris la reproduction) est assujettie à sa politique d'utilisation que vous pouvez consulter en ligne.

https://apropos.erudit.org/fr/usagers/politique-dutilisation/ 


\title{
La politique des salaires
}

\author{
Jacques St-Laurent \\ Marc-Adélard Tremblay \\ Yves Dubé
}

\section{Notion, objectifs, possibilités}

\section{Jacques St-Laurent}

Après avoir défini la notion de politique de salaires, l'auteur tente d'en expliquer les objectifs et d'identifier les moyens à employer pour la rendre efficace. Mais, en fait, on parle beaucoup de politique de salaires mais que fait-on concrètement à ce sujet?

\section{Définition}

Comme, dans l'esprit des gens, l'expression «politique de salaires 》 prend des sens différents, il convient, dès le départ, de préciser le sens que nous allons lui donner.

On peut donner à l'expression «politique de salaires » un sens plus ou moins large. Dans un sens très large, on pourrait tout simplement définir la politique de salaires comme toute politique qui influence le développement de ces derniers. Le terme incluerait alors toutes les politiques gouvernementales comme les politiques fiscales, les politiques monétaires, les politiques commerciales, la politique concernant le taux de change, les politiques concernant les combines etc., etc. Cette définition est évidemment beaucoup trop large puisque, au fond, elle implique qu'il faut considérer comme un élément d'une politique de salaires toute décision affectant l'offre ou la demande de travail dont l'interrelation établit les salaires.

Dans un sens moins large, on pourrait parler de la politique de salaires des employeurs, des syndi-

ST-LAURENT, Jacques, professeur. Département des relations industrielles, Université Laval, Québec. 
cats ou de celle des divers gouvernements ou organismes publiques vis-àvis leurs propres employés. Par exemple, un employeur pourrait décider de maintenir des salaires à un niveau légèrement supérieur à celui du marché afin de réduire le taux de roulement de sa main d'oeuvre, s'assurer qu'elle soit de meilleure qualité ou faciliter l'embauchage de nouveaux employés lorsque le besoin s'en fait sentir. Un syndicat pourrait viser à la parité des salaires sans se soucier des effets sur l'emploi d'une telle politique ou de satisfaire des taux locaux dans le but d'éviter à plus ou moins brève échéance une réduction dans l'emploi.

Dans un sens beaucoup plus strict, l'expression «politique de salaires > se rapporte au développement par le gouvernement, de critères ou de repères (guidelines) spécifiques concernant la fixation ou l'évolution des salaires et des mesures nécessaires à amener tous les employeurs et tous les employés à s'y soumettre. Dans ce sens, le gouvernement établit une politique de salaires non plus exclusivement à l'égard de ses propres employés, mais à l'intention de l'ensemble de l'économie. Une telle définition exclut les moyens indirects d'affecter les salaires (politique fiscale, politique monétaire, etc) pour ne retenir que ceux qui les affecte directement.

L'élaboration d'une telle politique de salaires implique deux choses: d'abord la définition de l'objectif poursuivi; ensuite, l'élaboration des moyens propres à le réaliser. Dans la définition de l'objectif comme dans l'élaboration des mesures propres à le réaliser, des difficultés importantes surgissent qu'il est utile de souligner.

\section{Objectifs}

Le problème de définir les objectifs de salaires appropriés pour toute une économie n'est pas une tâche facile, ni simple. En général, jusqu'ici l'élaboration de ces objectifs a impliqué soit des prévisions sur la tenue générale de l'économie pour l'année suivante, soit des prévisions sur l'évolution de la productivité.

Dans le premier cas, on risque de provoquer des discussions qui pourraient fort bien être passionnées sur la valeur des projections annuelles. De plus, si l'on veut être sérieux, les objectifs devront être revisés annuellement en regard de l'évolution de la situation économique. Cela conduit inévitablement un gouvernement à procéder à une planification de plus en plus poussée. Il est possible que la population et les agents économiques refusent de s'engager jusqu'au degré de planification requis pour que les objectifs formulés en matière de salaires soient sérieux. Dans cette alternative, les prévisions à court terme de la situation économique 
manqueront de précision et le gouvernement perdra la confiance du public dans l'élaboration de sa politique de salaires.

Dans le second cas, celui d'objectifs de salaires découlant de l'estimé des tendances de la productivité, deux problèmes majeurs se posent. D'abord, quelle doit être la durée des séries statistiques servant à établir la tendance de la productivité ? Ensuite, comment devrait-on calculer cette tendance? On peut prévoir à l'égard de ces deux problèmes de nombreux désaccords puisque le choix de la durée des séries statistiques et de la méthode utilisée affecteront la valeur apportée à la tendance de la productivité. C'est ainsi que, pour la période allant de 1946 à 1963, le Conseil économique du Canada a estimé que la production réelle par personne employée avait augmenté d'environ $50 \%$ et que la production réelle par homme-heure avait augmenté d'environ $70 \%{ }^{(1)}$, alors que pour la même période le Bureau fédéral de la statistique estimait que ces pourcentages étaient respectivement d'environ $68 \%$ et $90 \%{ }^{\left({ }^{2}\right)}$.

Même si ces difficultés techniques étaient surmontées, il demeure que les estimés peuvent être rapidement faussés par un changement des politiques gouvernementales autres que celle du salaires. De plus, la liaison de l'évolution des salaires à celle de la productivité implique qu'on est satisfait de la distribution actuelle du revenu national, ce qui n'est pas nécessairement le cas: les exigences de la croissance économique peuvent rendre nécessaire un changement dans cette distribution.

Supposons maintenant qu'on a surmonté toutes ces difficultés et que tout le monde s'entend sur le taux d'augmentation des salaires. Il reste le problème, dont la solution est encore plus difficile, d'apporter occasionnellement des modifications au taux d'augmentation afin de tenir compte du fait que l'évolution du marché du travail n'est pas la même dans toutes les occupations ou dans toutes les régions d'une province ou d'un pays. Pour beaucoup d'économistes, c'est ici que l'on rencontre la difficulté principale de l'application d'une politique de salaires.

En effet, la connaissance qu'on a des divers marchés de travail étant très mince, l'élaboration de critères de différenciation dans les taux d'augmentation de salaires est pratiquement impossible. Il apparaît que le mécanisme même du marché peut régler ce problème de meilleure façon que par le truchement d'un ensemble de règles ou de critères élaborés par un gouvernement.

(1) Objectifs économiques du Canada pour 1970, Premier exposé annuel du Conseil économique du Canada, Ottawa, décembre 1964.

(2) Bulletin hebdomadaire, Bureau fédéral de la statistique, 28 juillet 1967. 
En fait, il semble que l'on se trouve devant le dilemme suivant: ou on colle rigidement aux critères élaborés par le gouvernement et l'on aboutit à une allocation des ressources qui n'est pas nécessairement la meilleure puisque l'on ne tient pas compte de l'évolution différente des marchés de travail et du rôle que jouent des augmentations de salaires différenciées dans la satisfaction de cette évolution; ou l'on accepte des dérogations aux normes gouvernementales toutes les fois que l'exige une meilleure allocation des ressources et la politique de salaires se vide de son sens.

\section{Les moyens}

Supposons maintenant qu'on a réussi à établir des normes générales et (ou) particulières. Il s'agit maintenant de trouver les moyens de forcer les employeurs et les employés à y adhérer.

Le moyen le plus simple, mais aussi le plus faible, qu'on a utilisé jusqu'ici dans un certain nombre de pays, c'est celui de la persuasion. Ce moyen est généralement faible parce que les conditions nécessaires à son efficacité sont rarement réalisées. En effet, pour qu'il soit efficace il faut que le public appuie la mesure gouvernementale et que le nombre de ceux visés par ces mesures soit petit, car le petit nombre facilite l'identification rapide de ceux qui ne se conforment pas aux mesures gouvernementales, donc l'identification des responsables de l'échec de la politique gouvernementale ${ }^{(3)}$. Or, dans le cas d'une politique générale de salaires, la première condition peut n'être pas réalisée alors que la seconde l'est rarement.

Dans ces circonstances, le succès des mesures gouvernementales dépend de l'utilisation de moyens beaucoup plus efficaces mais aussi beaucoup plus drastiques. Le législateur doit alors intervenir pour rendre obligatoire l'adhérence aux mesures qui jusque-là n'étaient que proposées ou suggérées. Cependant, l'utilisation de la loi pour faciliter le respect d'une politique de salaires ne va pas sans difficulté. À cause de son impopularité, un tel geste n'est possible que dans les circonstances où il est évident pour tout le monde que l'intérêt de l'économie l'exige.

En effet, l'adhésion soutenue du public à une politique générale de salaires n'est possible que dans des circonstances particulières bien précises, par ailleurs désagréables, comme la désorganisation d'une économie à la suite d'une guerre (exemple: la politique de salaires aux Pays-Bas) ${ }^{\left({ }^{4}\right)}$

(3) J. T. Romans, « Moral Suasion as an Instrument of Economic Policy », dans American Economic Review, dec. 1966, p. 1220 sq.

(4) « The Strange Adventures of Dutch Wage Policy », dans British Journal of Industrial Relations, oct. 1963, pp. 318-330. 
ou une menace de désorganisation à la suite de problèmes chroniques dans la balance des paiements (exemple: les récentes mesures prises par l'Angleterre concernant les prix et les salaires). Dans toute autre circonstance, une législation à l'appui d'une politique de salaire court le risque de demeurer à l'état de projet.

Mais même dans les circonstances où une législation est possible, cette dernière doit être consistante avec les exigences du marché provincial ou interprovincial, national ou international du travail. Si elle ne l'est pas, on court à brève échéance le risque d'avoir un surplus ou une pénurie de main-d'oeuvre.

En effet, l'intégration économique à d'autres provinces ou à d'autres pays limite grandement la possibilité d'une politique indépendante des salaires. Le gouvernement dont on disait le plus que sa politique de salaires a été heureuse, celui des Pays-Bas, a dû faire face en 1964 à un choc généralement appelé «l'explosion des salaires», choc causé par le fait que les augmentations internes de salaires ne correspondaient pas aux exigences du marché international du travail ${ }^{(5)}$. Ces exigences compromettent singulièrement la réussite d'une politique de salaires.

Par ailleurs, même si on fait abstraction des problèmes externes d'une politique de salaire, l'expérience prouve que le respect interne de cette politique - si elle n'est pas conforme aux exigences du marché interne - est toujours compromis: les employeurs trouvent toujours « des moyens ingénieux pour contourner les règlements qui ne favorisent pas leurs intérêts ${ }^{(8)}$. Aux Pays-Bas, "les salaires du marché noir ont été, pendant un temps une particularité importante du régime ${ }^{(7)}$.

\section{Les faits}

Ce tableau des conditions de réalisation d'une politique de salaires contraste durement avec l'enthousiasme avec lequel il est aujourd'hui de mode de parler de politique de salaires de la part des gouvernements pour l'ensemble de l'économie dont ils sont responsables. Il demeure néanmoins que même une enquête rapide des politiques de salaires dans les différents pays de l'Ouest qui ont dit en avoir eu une révèle qu'à leur égard on a fait beaucoup plus de discours que de gestes. À la suite d'une étude sur les politiques de salaires et de prix dans quatre pays d'Europe, les auteurs

(5) J. J. KLOMT, «Holland after the Wage Explosion» dans The Banker, janvier 1965.

(6) David C. Smith, Politiques des revenus, Étude spéciale \# 4 préparée pour le Conseil économique du Canada, Ottawa, 1966, p. 161.

(7) Idem, p. 162. 
affirmaient ce qui suit: «a very high proportion of the legislation, speaches, bargaining, and even legal contracts revolving around these policies (i.e. wage and price policies) turn out to be significant for the symbolic reassurance they convey, for they frequently had little or no concrete impact upon wage or price levels $>{ }^{(8)}$.

Plus près de nous, et de façon générale, on a fortement mis en doute l'utilité des repères du président américain. À ce sujet, en avril '66, se réunissait à l'Université de Chicago un groupe d'économistes ${ }^{(9)}$. Seul, le conseiller économique du président, Gardner Ackley, a offert une défense, assez faible d'ailleurs, de la politique des repères. Avant même, disait-il, que soit atteint le plein emploi des ressources, des unions puissantes obtiennent des augmentations de salaires qui vont au-delà de l'augmentation de la productivité et des entreprises oligopolistiques augmentent leurs prix avec l'augmentation de la demande. C'est à l'intérieur de cette bande de pressions inflationnistes sur les coûts, avant même d'avoir atteint le plein emploi des ressources, que les repères sont proposés aux unions, aux chefs d'entreprises qui ont une certaine discrétion dans l'établissement des prix et des salaires et qu'on fait appel à leur sens des responsabilités pour s'y conformer.

Robert Solow lui, croit que les repères pourraient avoir une peu d'effet sur les prix sans que cela coûte très cher en faisant appel aux individus et aux organismes qui sont sensibles à l'opinion publique.

De son côté, monsieur John Dunlop, bien qu'il reconnaisse la valeur des préoccupations qui ont donné naissance aux repères, les conteste en affirmant qu'ils n'ont pas reçu un appui bien fort, ni de la part des syndicats, ni de la part des employeurs; qu'ils ne sont pas exprimés en termes opérationnels du point de vue de ceux qui ont à prendre des décisions (les médiateurs et les arbitres); et qu'ils sont inéquitables puisqu'on n'avait prévu aucune procédure de révision.

Enfin, Milton Friedman, beaucoup plus catégorique, affirme que les repères sont indésirables puisqu'ils distraient l'attention des causes fondamentales et que le fait qu'on ne s'y conforme qu'ici et là amène des distorsions dans les prix relatifs.

(8) M. Edelman et R. W. Fleming, The Politics of Wage-Price Decisions, A Four Country Analysis, University of Illinois Press, 1965 (Les 4 pays dont il est question sont les suivants: Italie, Allemagne, Pays-Bas et Grande-Bretagne).

(9) G. P. Shultz et R. Aliber, Ed., Guidelines, Informal Controls, and the Market Place: Policy Choices in a Full Employment Economy, The University of Chicago Press, 1966. 
On a tenté de ré-habiliter les repères (guidelines) américains en se demandant si l'augmentation des salaires (et des prix) n'aurait pas été plus importante sans eux. Tout ce qu'on a pu dire, c'est qu'ils «semblaient » avoir ralenti l'augmentation des salaires, mais qu'il était possible que d'autres facteurs en aient été la cause ${ }^{(10)}$.

\section{Conclusion}

Dans ces circonstances, comment expliquer la popularité des discussions sur une politique de salaires? Avec beaucoup de dureté, des auteurs ont affirmé que "when a large mass feels threatened by development over which it has little or no influence, it is likely to be easily manipulated and to be pleased by gestures that bring little tangible benefit but do serve as a reassurance ${ }^{(11)}$.

N'est-on pas alors en droit de se demander si les prises de position, les gestes et les discours sur la politique de salaires n'ont pas seulement pour but d'augmenter la liberté d'action, le statut ou le pouvoir politique de ceux qui en parlent?

\section{Les implications salariales des comportements de consommation}

\section{Marc-Adélard Tremblay}

L'auteur tente ici de traduire la manière dont le consommateur se conçoit lui-même, essaie de faire ressortir les pressions qui s'exercent sur ce dernier et définit les ressources dont il devrait disposer pour se procurer les biens et services qu'il juge nécessaires.

\section{Introduction}

Dans ce bref exposé, nous nous plaçons sous l'angle de l'indivi-
TREMBLAY, Marc-Adélard, Ph.D., professeur, Département de sociologie et d'anthropologie, Université Laval, Québec.

(10) G. L. Perry, «Wages and Guideposts », dans American Economic Review, sept. 1967 , p. 897 et sq.

(ii) M. Edelman et R. W. Fleming, op. cit., p. 309. 